\title{
Genetic and functional evidence relates a missense variant in B4GALT1 to lower LDL-C and fibrinogen
}

May E. Montasser ${ }^{1}$, Cristopher V.Van Hout ${ }^{2}$, Rebecca McFarland ${ }^{1}$, Avraham Rosenberg ${ }^{3}$, Myrasol Callaway ${ }^{3}$, Biao Shen ${ }^{3}$, Ning Li ${ }^{3}$, Thomas J. Daly ${ }^{3}$, Alicia D. Howard ${ }^{1}$, Wei Lin ${ }^{3}$, Yuan $\mathrm{Mao}^{3}$, Bin Ye ${ }^{2}$, Giusy Della Gatta ${ }^{2}$, Gannie Tzoneva ${ }^{2}$, James Perry ${ }^{1}$, Kathleen A. Ryan ${ }^{1}$, Lawrence Miloscio ${ }^{2}$, Aris N. Economides ${ }^{2}$, Regeneron Genetics Center ${ }^{4}$, NHLBI TOPMed Program $^{4}$, Carole Sztalryd-Woodle ${ }^{1}$, Braxton D. Mitchell ${ }^{1,5}$, Matthew Healy ${ }^{2}$, Elizabeth Streeten ${ }^{1}$, Norann A. Zaghloul ${ }^{1}$, Simeon I. Taylor ${ }^{1}$, Jeffrey R. O'Connell ${ }^{1}$, Alan R. Shuldiner ${ }^{2}$

${ }^{1}$ Program for Personalized and Genomic Medicine, Department of Medicine, University of Maryland School of Medicine, Baltimore, Maryland, USA

${ }^{2}$ Regeneron Genetics Center, LLC., Tarrytown, NY 10591, USA

${ }^{3}$ Regeneron Pharmaceuticals, Inc., Tarrytown, NY 10591, USA

${ }^{4}$ Full list of names and affiliations in the online supplementary materials

${ }^{5}$ Geriatrics Research and Education Clinical Center, Baltimore VA Medical Center, Baltimore, MD 21201, USA 


\section{Abstract}

Increased LDL-cholesterol (LDL-C) and fibrinogen are independent risk factors for cardiovascular disease (CVD). We identified novel associations between an Amishenriched missense variant (p.Asn352Ser) in a functional domain of beta-1,4galactosyltransferase 1 (B4GALT1) and $13.5 \mathrm{mg} / \mathrm{dl}$ lower LDL-C ( $p=1.6 \mathrm{E}-15)$, and $26 \mathrm{mg} / \mathrm{dl}$ lower plasma fibrinogen ( $p=9.8 E-05)$. N-linked glycan profiling found $p$.Asn352Ser to be associated (p-values from 1.4E-06 to $1.0 \mathrm{E}-17$ ) with decreased glycosylation of glycoproteins including: fibrinogen, ApoB100, immunoglobulin G (lgG), and transferrin. In vitro assays found that the mutant (352Ser) protein had $50 \%$ lower galactosyltransferase activity compared to wild type (352Asn) protein. Knockdown of b4galt1 in zebrafish embryos resulted in significantly lower LDL-C compared to control, which was fully rescued by co-expression of 352Asn human B4GALT1 mRNA but only partially rescued by co-expression of 352Ser human B4GALT1 mRNA. Our findings establish B4GALT1 as a novel gene associated with lower LDL-C and fibrinogen and suggest that targeted modulation of protein glycosylation may represent a therapeutic approach to decrease CVD risk.

Cardiovascular disease (CVD) accounts for 1 of every 3 deaths in the USA and is the leading cause of morbidity and mortality worldwide ${ }^{1}$. Elevated low-density lipoprotein cholesterol (LDL-C) increases arterial plaque formation and atherosclerosis, and fibrinogen increases risk for blood clotting and thrombosis; both are independent risk factors for coronary artery disease $(C A D)^{2,3}$. LDL-C and fibrinogen are governed by both genetic and environmental factors, as well as the interplay between them ${ }^{4,5}$. Rare variants in LDLR, PCSK9, APOB cause severe forms of familial hypercholesterolemia, and many common genetic variants, each with small effect size, have been identified in large genome-wide association studies of both LDL-C and fibrinogen ${ }^{6-8}$. However, few variants have been found with pleiotropic effects on more than one CAD risk factor. Similarly therapeutic approaches to mitigate CAD risk have focused on treating individual risk factors. Deeper understanding of the genetic determinants of LDL-C and fibrinogen may unveil novel targets for therapy that may be more efficacious and safe to treat or prevent CAD.

Founder populations can facilitate the identification of novel disease associations with variants that are enriched to a higher frequency through genetic drift. Multiple examples have been recently reported of highly enriched variants with large effect sizes associated with complex diseases and traits in homogenous populations in Iceland ${ }^{9}$, Sardinia ${ }^{10}$, Greenland ${ }^{11}$, Samoa $^{12}$ and the Old Order Amish (OOA) ${ }^{13-19}$. While such drifted variants are often rare or absent in the general population, their novel associations can inform biological mechanisms and therapeutic targets relevant to all humans.

The ability to perform whole-exome and whole-genome sequencing in founder populations provides the opportunity to identify drifted causative genetic variants hard to identify in general populations. In this study, we performed genetic association analyses for LDL-C in OOA participants with whole-genome and whole-exome sequencing data. We identified a novel genome-wide significant association between an Amish-enriched missense variant in the beta1,4-galactosyltransferase 1 gene (B4GALT1 p.Asn352Ser) and decreased LDL-C, which was also associated with decreased fibrinogen. Homozygotes for $352 \mathrm{Ser}$ have increased levels of incompletely synthesized glycans on glycoproteins as a result of decreased B4GALT1 enzymatic activity suggesting a potential mechanism to modulate lipid metabolism and coagulation. 


\section{Association analyses identify B4GALT1 p.Asn352Ser as a novel LDL-C variant}

To identify genetic variants associated with LDL-C, we performed an exome-wide association scan using 5,890 OOA subjects with whole-exome sequencing (WES) performed at the Regeneron Genetics Center. Demographic and clinical characteristics of the WES subjects are shown in Supplementary Table 1. Linear mixed model association analysis identified several previously known loci for LDL-C as well as a novel locus on the short arm of chromosome 9 (Supplementary Fig. 1 and Supplementary Table 2). As shown in Figure 1a, a missense variant (rs551564683, p.Asn352Ser) in B4GALT1 was strongly associated with $13.5 \mathrm{mg} / \mathrm{dl}$ lower LDL-C in an additive genetic model ( $p=1.6 \mathrm{E}-15)$. This variant has a minor allele frequency (MAF) of $6 \%$ in the OOA population while extremely rare in the general population (only 8 copies were identified in 140,000 WGS of non-Amish participants in the NHLBI Trans-Omics for Precision Medicine (TOPMed) program (www.nhlbiwgs.org).

Since WES captures only the coding variants, we performed association analysis using 1,083 OOA subjects with whole-genome sequence (WGS) as part of TOPMed (dbGaP accession number: phs000956) to exhaustively interrogate all the coding and noncoding genetic variants in this region. Despite the much smaller sample size, the WGS analysis identified the same B4GALT1 variant rs551564683 as the top signal in this region with p-value of $3.1 \mathrm{E}-06$ and effect size of $16.9 \mathrm{mg} / \mathrm{dl}$ lower LDL-C (Figure $1 \mathrm{~b}$ ). In addition, WGS analysis revealed 20 variants in the region in high linkage disequilibrium (LD) with rs551564683 $\left(r^{2}>0.8\right.$, range from 0.84 to 0.95 ), with LDL-C association p-values ranging from 6.3E-06 to 2.3E-05 (Supplementary Table 3). Of the 21 variants, rs551564683 is the only protein coding variant (B4GALT1 p.Asn352Ser), and is classified as damaging or deleterious by 5 in silico protein function prediction algorithms (SIFT = deleterious, Polyphen2 = possibly damaging, LRT = deleterious, Mutation Taster $=$ disease-causing, PROVEAN = deleterious). These 20 variants and rs551564683 comprise a $4 \mathrm{Mb}$ Amish-specific haplotype extending from approximately $31.5 \mathrm{Mb}$ to $35.5 \mathrm{Mb}$ on chromosome 9 (Supplementary Fig. 2). These variants have a MAF of approximately $6 \%$ in the OOA while extremely rare or nonexistent in the general population (Supplementary Table 3)

The limited sample size of the WGS $(n=1,083)$ was not able to differentiate statistically the top missense variant ( $p=3.1 \mathrm{E}-06$ ) from the 20 other highly correlated variants ( $p$ from 6.3E06 to 2.3E-05). To further differentiate between these 21 highly linked variants, we imputed genotypes in the full OOA dataset of 5,890 subjects with genotype chip data to the TOPMed WGS reference panel. As shown in Figure 1c and Supplementary Table 2, the missense variant was the top associated variant with a $p$ value of $3.6 \mathrm{E}-15$, two or more orders of magnitude smaller than any of the other variants ( $p$ from $9.4 \mathrm{E}-13$ to $1.6 \mathrm{E}-09$ ). Independent direct genotyping for 7 of these variants gave similar results (data not shown).

To determine if rs551564683 is the sole signal in the region, conditional analysis was performed. Conditional analysis adjusting for rs551564683 completely abolished the association of the other 20 variants while conditional analyses adjusting for any of the other 20 variants reduced the association of rs551564683 due to the strong correlation $\left(r^{2}=0.84-0.95\right)$, but did not abolish it ( $p=1.0 \mathrm{E}-03-3.0 \mathrm{E}-07$ ). Our analysis supports rs551564683 as the most likely causal variant in this region.

\section{Association with other traits and B4GALT1 human knockout support a functional role of B4GALT1 p.Asn352Ser}


$B 4 G A L T 1$ is a member of the beta-1,4-galactosyltransferase gene family which encode type II membrane-bound glycoprotein that plays a critical role in the processing of $\mathrm{N}$-linked oligosaccharide moieties in glycoproteins. Impairment of B4GALT1 activity has the potential to alter the structure of $\mathrm{N}$-linked oligosaccharides and introduce aberrations in glycan structure that have the potential to alter glycoprotein function.

A homozygous frame shift insertion in B4GALT1 that results in a truncated dysfunctional protein was previously reported to cause Congenital Disorder of Glycosylation type 2 (CDGII) in 2 patients ${ }^{20,21}$. These 2 patients exhibited, among other traits, abnormal coagulation and very high levels of aspartate transaminase (AST). Interestingly we found B4GALT1 p.Asn352Ser to be strongly associated with lower levels of fibrinogen $(N=805$, beta $=-26 \mathrm{mg} / \mathrm{dl}, \mathrm{p}=9.8 \mathrm{E}-05)$. We also found that the mean level of AST in 352Ser homozygotes was two-fold higher than that of 352Asn homozygotes (35.8 vs $18.5 \mathrm{U} / \mathrm{L}$, respectively) $(\mathrm{N}=5,595$, additive model $\mathrm{p}=2.3 \mathrm{E}-11$, recessive model $p=2.4 \mathrm{E}-25)$, further supporting the functional role of this variant. While the high level of AST might suggest liver injury, we found no association with alanine aminotransferase $(A L T)$, alkaline phosphatase (ALP), liver to spleen density ratio, or inflammatory markers (Supplementary Table 4). Moreover, all 352Ser homozygotes $(\mathrm{N}=12)$ had normal levels of gamma-glutamyl transpeptidase (GGT), activated partial thromboplastin time (aPTT), prothrombin time (PT), and internationalized normalized ratio (INR) (Supplementary Table 5).

\section{B4GALT1 p.Asn352Ser is associated with increased levels of incompletely synthesized glycans on glycoproteins}

To assess the impact of B4GALT1 p.Asn352Ser on glycosylation, the carbohydrate deficient transferrin (CDT) test, which assesses glycosylation levels of transferrin and apolipoprotein CIII (ApoCIII), was performed using serum samples from 28 participants from the 3 genotype groups (9 352Ser homozygotes, 8 Asn352Ser heterozygotes and 11 352Asn homozygotes). As expected, based on the fact that B4GALT1 plays a role in processing of Nlinked carbohydrate, all samples had normal profiles for ApoCIII O-linked carbohydrate (Supplementary Table 6). All samples also had normal levels of mono-oligosaccharide/dioligosaccharide transferrin ratio, and a-oligosaccharide/di-oligosaccharide transferrin ratio. However, while all eight 352Asn homozygotes had normal levels of the tri-sialo/dioligosaccharide transferrin ratio, the ratio in all 352Ser homozygotes was abnormal; heterozygotes were intermediate between the two homozygote groups ( $p=9.12 \mathrm{E}-10)$ (Figure 2 and Supplementary Table 6). Since transferrin is mostly tetrasialylated ${ }^{22,23}$, this increase in trisialylated transferrin reflects a paucity of tetrasialylated molecules and indicates that the 352 Ser allele is associated with increased levels of carbohydrate deficient transferrin, likely due to decreased enzymatic activity of B4GALT1.

To determine if the lower levels of sialylation are affecting only transferrin or extending to other glycoproteins, N-linked glycan profiling was performed using plasma samples from 24 participants (12 352Ser homozygotes and 12 352Asn homozygotes) for global plasma glycoproteins, as well as selected specific plasma glycoproteins - apolipoprotein B-100 (ApoB100), fibrinogen and immunoglobulin G (IgG) - by Hydrophilic Interaction Liquid Chromatography with Fluorescent Detection and Mass Spectroscopy (HILIC-FLR-MS).

Glycans from 352Ser homozygotes had increased levels of incompletely synthesized oligosaccharides as evidenced by increased percentages of truncated biantennary glycans devoid of galactoses and sialic acids (GOF, $p=5.4 \mathrm{E}-11$; $b \mathrm{G} 0, \mathrm{p}=2.1 \mathrm{E}-6)$, and biantennary glycans with only one galactose and one sialic acid (G1S1, $p=5.1 \mathrm{E}-16)$. Reciprocally, 352Ser homozygotes had significantly lower levels of biantennary glycans with two galactose and two 
sialic acid residues (G2S2, $\mathrm{p}=2.6 \mathrm{E}-8$ ) (Supplementary Table 7). The results for plasma ApoB100, fibrinogen and IgG were similar, where serum from 352Ser homozygotes had significantly increased level of glycans lacking galactose and sialic acid moieties and decreased levels of more mature glycans (Supplementary Table 8, 9, 10).

Overall, there was significantly lower galactosylation ( $p$ from $1.8 \mathrm{E}-08$ to $1.0 \mathrm{E}-17$ ) and sialylation ( $p$ from 1.4E-06 to 5.6E-15) for global glycoproteins, and enriched ApoB100, fibrinogen and IgG among 352Ser homozygotes compared to 352Asn homozygotes, however there was no difference in fucosylation (Figure 3), which is consistent with the role of B4GALT1 in adding galactose moieties that then are capped by sialic acid while having no role in the addition of fucose ${ }^{24}$.

In summary, the 352 Ser allele is associated with significantly increased levels of incompletely synthesized glycans on glycoproteins indicating defective protein glycosylation.

\section{B4GALT1 p.Asn352Ser causes reduced enzymatic activity}

To compare the in vitro enzymatic activities of wild type (352Asn) and mutant (352Ser) B4GALT1 protein, we transiently transfected COS-7 cells, which express very low endogenous levels of B4GALT1, with cDNAs encoding myc-FLAG epitope-tagged versions of the proteins. We immunoprecipitated with anti-FLAG antibody, and measured B4GALT1 activities in the immune complexes (Supplementary Fig. 3). Compared to 352Asn B4GALT1, 352Ser B4GALT1 showed on average $50 \%$ decrease in galactosyltransferase specific activity (Figure 4a).

As a complementary approach, we used synthesized recombinant human 352Asn and 352Ser B4GALT1 protein to test the reduction rate of the substrate uridine diphosphate galactose (UDP-Gal) incubated with each protein and found that the UDP-Gal level decreased faster with 352Asn than 352Ser B4GALT1, indicating decreased enzymatic activity of 352Ser B4GALT1 compared to 352Asn B4GALT1 (Figure 4b).

\section{Functional validation of the effect of B4GALT1 p.Asn352Ser on LDL-C in zebrafish}

We used a zebrafish model to investigate the effect of B4GALT1 p.Asn352Ser on LDL-C using our established assays ${ }^{19,25}$. We first generated a genomic knockout of the zebrafish ortholog (b4galt1) using CRISPR/Cas9-mediated targeting of exon 2. Consistent with mouse reports of embryonic lethality in knockout animals ${ }^{26}$ injected F0 animals were not viable to adulthood and consistently died at juvenile stages. To circumvent the lack of viability, we employed a knockdown approach using a previously reported splice-blocking antisense morpholino oligonucleotide (MO) injected into embryos ${ }^{27}$. After validation of MO efficacy (Supplementary Fig. 4) and ruling out the possibility of off-target toxicity by demonstrating no increases in d113 p53 expression and no overt morphological defects (Supplementary Fig. 5, $6 a$ ), we quantified changes in LDL-C levels in unfed larvae at 5 days post fertilization (dpf) as per previously published protocols ${ }^{25}$. We observed a significant decrease in LDL-C in MOinjected larvae compared to control larvae, consistent with a role for b4galt1 in LDL-C homeostasis (Figure 5). We further confirmed this result using a second splice-blocking MO targeting exon 2 of b4galt 1 which similarly produced a reduction in LDL-C concentration (Supplementary Fig. 6b-d).

To validate the specificity of these observations and to test the functionality of human B4GALT1 in zebrafish, we co-injected mRNA encoding human 352Asn B4GALT1 along with 
b4galt1 $\mathrm{MO}$ into embryos and assessed LDL-C in unfed larvae. This resulted in LDL-C levels that were statistically indistinguishable from those in larvae injected only with a control MO $(p=0.45)$, suggesting that the human mRNA could rescue the effects of knockdown of the zebrafish gene (Figure 5).

These data suggest that human wild type B4GALT1 mRNA is functional in zebrafish and support the use of this model system for functional interpretation of p.Asn352Ser. Using sitedirected mutagenesis ${ }^{28}$, we introduced a $T$ to $C$ change in the coding sequence of human B4GALT1 and generated full length mRNA. Co-injection of the 352Ser B4GALT1 mRNA with b4galt1 $\mathrm{MO}$ resulted in a reduced capacity for rescue of the LDL-C phenotype. The resulting LDL-C concentration was $25 \%$ lower than that resulting from co-injection of 352Asn B4GALT1 mRNA with $b 4 g$ alt1 $\mathrm{MO}$, a statistically significant effect $(\mathrm{p}=0.03)$. This level of LDL-C was also statistically greater, however, than b4galt1 $\mathrm{MO}$ alone $(\mathrm{p}=0.02)$ (Figure 5$)$, suggesting only a partial defect in function introduced by the missense variant.

To examine the relevance of b4galt 1 to other hypercholesterolemic models, we examined the impact of b4galt1 knock down on two previously reported zebrafish models of elevated LDL-C: high cholesterol diet fed larvae and $/ d l r$ knockdown animals ${ }^{25}$. For the former, we treated $5 \mathrm{dpf}$ larvae with either a control diet or the same diet supplemented with $4 \% \mathrm{w} / \mathrm{w}$ cholesterol as previously reported ${ }^{25}$. While b4galt1 knockdown reduced LDL-C in animals fed a control diet, this effect was abolished with introduction of a high cholesterol diet (Supplementary Fig. 7). In contrast, when we introduced b4galt1 suppression on a background of geneticallyinduced hypercholesterolemia via Idlr MO injection, we observed amelioration of the LDL-C phenotype in those animals (Supplementary Fig. 7). These data suggest that suppression of b4galt 1 can abrogate elevated LDL-C resulting from genetic defects in LDL-C metabolism but not that introduced by diet.

\section{Discussion}

Large genome-wide analyses of approximately 600,000 individuals have identified 386 loci associated with lipid traits, none of which identified the B4GALT1 gene ${ }^{29}$. Using next generation sequencing in 5,890 OOA, we found a strong novel association of B4GALT1 p.Asn352Ser with lower plasma LDL-C and fibrinogen levels. Glycosylation profiling and experimental assays confirmed the functional role of this variant. This variant is very rare in the general population, but has $6 \%$ frequency in the Amish, likely due to genetic drift over approximately 14 generations after founding. This report highlights the value of founder populations in identifying novel gene variants that can provide new insights into human biology of common traits.

Homozygosity for a protein truncating mutation in B4GALT1 (1032insC) reported in two related patients is known to cause CDG type 2 (CDGII) ${ }^{20,21}$. Both patients have marked abnormal carbohydrate structures on glycosylated proteins and severe clinical phenotypes manifesting in early childhood with developmental delay, hypotonia, coagulopathy, and elevated transaminases ${ }^{20,21,30}$. Knock-out of $b 4$ galt 1 in mice results in semi-lethality after birth and several other sever developmental abnormalities ${ }^{31,32}$. Interestingly, mass spectrometry studies of $\mathrm{N}$ glycans from plasma and hepatic membrane glycoproteins, revealed an unexpected high level of sialylation and galactosylation in b4galt $1-/-(\sim 60 \%$ compared to that of b4galt $1+/+$ mice $)$. Kotani et. al., explained the altered pattern of sialylation and galactosylation with a shift in synthesis from type 2 to type 1 glycan chains in $b 4 g a l t 1^{-/-}$versus $b 4 g a l t 1^{+/+}$mice, most probably caused by b3galt proteins in response to loss of $b 4$ galt $1^{33}$. Conversely, the overall decrease in galactosylation and sialylation that we observed in the carriers of 352Ser B4GALT1 missense 
variant, suggests that this missense variant could act as hypomorph. Indeed, our preliminary investigation showed that the level of type 1 chain (corresponding to the $b$ 1,3 linkage) was very low and comparable between 352Asn and 352Ser homozygotes (data not shown), suggesting that our missense mutation did not cause any glycan chain type switch as shown in Kotani et. al., and that compensatory action of B3GALT1 may differ between mouse and human.

The p.Asn352Ser B4GALT1 missense variant that we identified in the Amish does not appear to be associated with any severe phenotype. In fact, the self-reported medical and family history of fifteen 352Ser homozygotes did not note previous heart attack, stroke, coronary angiogram, blocked arteries, or heart/carotid artery surgery. None had evidence of coronary ischemia or MI on EKG except for one 70 year old person who showed evidence of a potential previous MI. Abdominal ultrasound from five 352Ser homozygotes over 50 years old showed normal abdominal aorta with no atherosclerotic plaque, fatty liver or aneurysm. The variant showed no association with reduced coronary artery calcification (CAC) or aortic calcification (AC), however, the sample size was small with relatively young average age of 52 , that included only one 352Ser homozygote (Supplementary Table 4).

We also found that 352 Ser homozygotes had double the serum AST levels compared to 352Asn homozygotes, however, they all had normal levels of ALT, GGT and ALP, suggesting that the source of high AST is from tissue other than liver, i.e., muscle. Moreover, they also had normal INR, PTT and inflammation markers (Supplementary Tables 4 and 5). The absence of a severe phenotype in the minor homozygotes may be due to the subtle effect of the missense mutation compared to the protein truncating frameshift mutation identified in CDGII patients.

The missense variant p.Asn352Ser changes the asparagine to serine at position 352 in the 398 amino acid sequence of the long isoform of human B4GALT1 (corresponding to position 311 of the short isoform). The asparagine residue is completely conserved across 100 vertebrate species (GERP score $=5.9$ ). p.Asn352Ser is located in the highly flexible long $C$ terminal region of the protein that undergoes conformational changes to allow for the exchange of the sugar molecule during glycosylation ${ }^{34}$. Hence, a mutation in this region may impede the necessary conformational change and impact glycosylation efficiency as shown by our glycosylation profiling and functional assays. However, it is important to note that the $352 \mathrm{Ser}$ mutation had less severe effects on glycosylation than that previously reported in CDGII patients carrying a B4GALT1 truncating mutation ${ }^{20}$.

The mechanism by which this variant leads to lower LDL-C and fibrinogen remains to be elucidated. B4GALT1 is a ubiquitously expressed protein that transfers the galactose from uridine diphosphate galactose (UDP-Gal) to specific glycoprotein substrates ${ }^{21}$. A defect in $B 4 G A L T 1$ that affects glycosylation and sialylation may affect the folding, secretion, stability, activity, and half-life of lipoprotein and coagulation related glycoproteins ${ }^{35-37}$ ultimately resulting in lower levels of circulating LDL-C and fibrinogen.

Genome-wide association studies (GWAS) have identified common non-coding variants at the B4GALT1 locus that are significantly associated with $\lg$ glycosylation and AST levels ${ }^{24,38}$, but not with any lipid traits. However, several lines of evidence point to protein glycosylation as a major player in lipid metabolism. Reduced APOB glycosylation leads to shorter LDL-C half-life and rapid clearance from the circulation, which in turn reduces its atherogenic effect ${ }^{35}$, while increased LDL-C glycosylation was associated with increased LDL-C oxidation leading to greater atherosclerosis ${ }^{39}$. Glucose mediated N-glycosylation plays a major role in regulating sterol regulatory element-binding proteins (SREBPs) which are major players in cholesterol metabolism ${ }^{40}$. Also, reduced LDLR glycosylation was found to result in reduced lipid binding and endocytosis ${ }^{41}$. Recently, changes in IgG glycosylation have been associated 
with blood lipids and dyslipidemia in Han Chinese ${ }^{42}$. A similar role for glycosylation was also reported in relation to coagulation proteins, where mutations affecting glycosylation were associated with different enzymatic activity ${ }^{37}$. Finally, GWAS has identified loci containing other enzymes involved in glycoprotein biology associated with lipid traits including GALNT2 ${ }^{43}$, ST3GAL4 ${ }^{6}$, and ASGR $1^{44}$.

Similarly, studies support a role of glycosylation in CVD. For example, a loss of function mutation in ASGR1 was reported to be associated with lower non-HDL cholesterol and reduced coronary artery disease (CAD) risk ${ }^{44}$. ASGR1 encodes a subunit of the asialoglycoprotein receptor (the "Ashwell receptor") that mediates binding and endocytosis of asialylated Nglycoproteins. Recently Menni et.al. ${ }^{45}$ reported that the glycosylation profile of IgG was associated with CVD risk score and subclinical atherosclerosis in two independent cohorts. While these studies are not specific to $B 4 G A L T 1$, they support an important role of glycosylation in CVD with potential prognostic and/or therapeutic implications.

In summary, we discovered a novel missense variant in B4GALT1 that is associated with lower LDL-C and fibrinogen, both cardioprotective phenotypes, through enrichment in a founder population. Evidence from human data as well as in vitro and animal-based experiments indicate that the variant leads to decreased protein glycosylation. Further understanding of the underlying biological mechanism of the variant may provide new insights into the role of glycosylation in CVD risk that may lead to novel therapeutic targets.

\section{Online content}

Methods, additional references, Nature Research reporting summaries, statements of data availability and associated accession codes are available in the online paper

\section{Acknowledgements}

This work was supported in part from NIH grants U01 HL137181, U01 HL072515, R01 AG18728, R01 HL121007, P30 DK072488, AHA 17GRNT33661168 and Regeneron Pharmaceuticals, Inc.

Whole-genome sequencing (WGS) for the Trans-Omics in Precision Medicine (TOPMed) program was supported by the National Heart, Lung and Blood Institute (NHLBI). WGS for "NHLBI TOPMed: Genetics of Cardiometabolic Health in the Amish" (phs000956) was performed at the Broad Institute of MIT and Harvard (HHSN268201500014C). Centralized read mapping and genotype calling, along with variant quality metrics and filtering were provided by the TOPMed Informatics Research Center (3R01HL-117626-02S1). Phenotype harmonization, data management, sample-identity QC, and general study coordination, were provided by the TOPMed Data Coordinating Center (3R01HL-120393-02S1).

We gratefully thank the Amish community and research volunteers for their longstanding partnership in research, and acknowledge the dedication of the Amish liaisons, field workers and the Amish Research Clinic staff, without which these studies would not have been possible.

\section{Author contributions}

Conceived, designed and supervised the work: MEM, ARS, ANE

Performed data collection: MEM, ARS, JO, BS, NL, GDG, GT, LM, AR, RMc, NAZ, WL, YM 
Contributed to data analysis MEM, CVH, BS, NL, BY, GDG, GT, LM, MH, JRO, RMc, NAZ, KAR, JP

Interpreted the results: MEM, ARS, CVH, BS, NL, TJD, GDG, ANE, MH, JRO, AH, CS, BDM, ES, NAZ, SIT

Preparation of the manuscript: MEM, ARS

\section{Competing interests}

CVH, GDG, AR, MC, TJD, BS, NL, BY, GT, LM, AE, MH, and ARS are current or former employees of Regeneron Pharmaceuticals Inc. MEM, CVH, ARS, GDG, and MH are inventors on a patent that was published by the United States Patent and Trademark Office on December 6, 2018 under Publication Number US 2018-0346888, and international patent application that was published on December 13, 2018 under Publication Number WO-2018/226560 regarding B4GALT1 Variants And Uses Thereof.

\section{Corresponding author}

Correspondence and requests for materials should be addressed to mmontass@som.umaryland.edu 


\section{References}

1. Mozaffarian D, Benjamin EJ, Go AS, et al. Heart disease and stroke statistics-2015 update: A report from the american heart association. Circulation 2015;131(4):e29-322.

2. Nelson $\mathrm{RH}$. Hyperlipidemia as a risk factor for cardiovascular disease. Prim Care 2013;40(1):195-211.

3. Meade TW. Fibrinogen and cardiovascular disease. J Clin Pathol 1997;50(1):13-15.

4. Lange LA, Hu Y, Zhang H, et al. Whole-exome sequencing identifies rare and low-frequency coding variants associated with LDL cholesterol. Am J Hum Genet 2014;94(2):233-245.

5. Su S, Snieder H, Miller AH, et al. Genetic and environmental influences on systemic markers of inflammation in middle-aged male twins. Atherosclerosis 2008;200(1):213-220.

6. Surakka I, Horikoshi M, Magi R, et al. The impact of low-frequency and rare variants on lipid levels. Nat Genet 2015;47(6):589-597.

7. Hoffmann TJ, Theusch E, Haldar T, et al. A large electronic-health-record-based genomewide study of serum lipids. Nat Genet 2018;50(3):401-413.

8. de Vries PS, Chasman DI, Sabater-Lleal M, et al. A meta-analysis of 120246 individuals identifies 18 new loci for fibrinogen concentration. Hum Mol Genet 2016;25(2):358-370.

9. Helgadottir A, Gretarsdottir S, Thorleifsson G, et al. Variants with large effects on blood lipids and the role of cholesterol and triglycerides in coronary disease. Nat Genet 2016;48(6):634639.

10. Sidore C, Busonero F, Maschio A, et al. Genome sequencing elucidates sardinian genetic architecture and augments association analyses for lipid and blood inflammatory markers. Nat Genet 2015;47(11):1272-1281.

11. Moltke I, Grarup N, Jorgensen ME, et al. A common greenlandic TBC1D4 variant confers muscle insulin resistance and type 2 diabetes. Nature 2014;512(7513):190-193.

12. Minster RL, Hawley NL, Su CT, et al. A thrifty variant in CREBRF strongly influences body mass index in samoans. Nat Genet 2016;48(9):1049-1054.

13. Pollin TI, Damcott CM, Shen $\mathrm{H}$, et al. A null mutation in human APOC3 confers a favorable plasma lipid profile and apparent cardioprotection. Science 2008;322(5908):1702-1705.

14. Shen H, Damcott CM, Rampersaud E, et al. Familial defective apolipoprotein B-100 and increased low-density lipoprotein cholesterol and coronary artery calcification in the old order amish. Arch Intern Med 2010;170(20):1850-1855.

15. Horenstein RB, Mitchell BD, Post WS, et al. The ABCG8 G574R variant, serum plant sterol levels, and cardiovascular disease risk in the old order amish. Arterioscler Thromb Vasc Biol 2013;33(2):413-419.

16. Daley E, Streeten EA, Sorkin JD, et al. Variable bone fragility associated with an amish COL1A2 variant and a knock-in mouse model. J Bone Miner Res 2010;25(2):247-261. 
17. Albert JS, Yerges-Armstrong LM, Horenstein RB, et al. Null mutation in hormone-sensitive lipase gene and risk of type 2 diabetes. N Engl J Med 2014;370(24):2307-2315.

18. Welty FK, Ordovas J, Schaefer EJ, Wilson PW, Young SG. Identification and molecular analysis of two apoB gene mutations causing low plasma cholesterol levels. Circulation 1995;92(8):2036-2040.

19. Montasser ME, O'Hare EA, Wang X, et al. An APOO pseudogene on chromosome $5 q$ is associated with LDL-C levels. Circulation 2018;138(13):1343-1355.

20. Guillard M, Morava E, de Ruijter J, et al. B4GALT1-congenital disorders of glycosylation presents as a non-neurologic glycosylation disorder with hepatointestinal involvement. $J$ Pediatr 2011;159(6):1041-3.e2.

21. Hansske B, Thiel C, Lubke T, et al. Deficiency of UDP-galactose:N-acetylglucosamine beta1,4-galactosyltransferase I causes the congenital disorder of glycosylation type Ild. $J$ Clin Invest 2002;109(6):725-733.

22. Helander A, Husa A, Jeppsson JO. Improved HPLC method for carbohydrate-deficient transferrin in serum. Clin Chem 2003;49(11):1881-1890.

23. Kranz C, Ng BG, Sun L, et al. COG8 deficiency causes new congenital disorder of glycosylation type IIh. Hum Mol Genet 2007;16(7):731-741.

24. Lauc G, Huffman JE, Pucic M, et al. Loci associated with N-glycosylation of human immunoglobulin $\mathrm{G}$ show pleiotropy with autoimmune diseases and haematological cancers. PLoS Genet 2013;9(1):e1003225.

25. O'Hare EA, Wang X, Montasser ME, Chang YP, Mitchell BD, Zaghloul NA. Disruption of Idlr causes increased LDL-C and vascular lipid accumulation in a zebrafish model of hypercholesterolemia. J Lipid Res 2014;55(11):2242-2253.

26. Stanley P. What have we learned from glycosyltransferase knockouts in mice? J Mol Biol 2016;428(16):3166-3182.

27. Machingo QJ, Fritz A, Shur BD. A beta1,4-galactosyltransferase is required for convergent extension movements in zebrafish. Dev Biol 2006;297(2):471-482.

28. O'Hare EA, Yang R, Yerges-Armstrong LM, et al. TM6SF2 rs58542926 impacts lipid processing in liver and small intestine. Hepatology 2017;65(5):1526-1542.

29. Klarin D, Damrauer SM, Cho K, et al. Genetics of blood lipids among 300,000 multi-ethnic participants of the million veteran program. Nat Genet 2018;50(11):1514-1523.

30. Perez-Cerda C, Giros ML, Serrano M, et al. A population-based study on congenital disorders of protein $\mathrm{N}$ - and combined with O-glycosylation experience in clinical and genetic diagnosis. J Pediatr 2017;183:170-177.e1.

31. Asano M, Furukawa K, Kido M, et al. Growth retardation and early death of beta-1,4galactosyltransferase knockout mice with augmented proliferation and abnormal differentiation of epithelial cells. EMBO J 1997;16(8):1850-1857.

32. Lu Q, Hasty P, Shur BD. Targeted mutation in beta1,4-galactosyltransferase leads to pituitary insufficiency and neonatal lethality. Dev Biol 1997;181(2):257-267. 
33. Kotani N, Asano M, Iwakura Y, Takasaki S. Knockout of mouse beta 1,4galactosyltransferase-1 gene results in a dramatic shift of outer chain moieties of $\mathrm{N}$-glycans from type 2 to type 1 chains in hepatic membrane and plasma glycoproteins. Biochem $\mathrm{J}$ 2001;357(Pt 3):827-834.

34. Qasba PK, Ramakrishnan B, Boeggeman E. Structure and function of beta -1,4galactosyltransferase. Curr Drug Targets 2008;9(4):292-309.

35. Filipovic I, Schwarzmann G, Mraz W, Wiegandt H, Buddecke E. Sialic-acid content of lowdensity lipoproteins controls their binding and uptake by cultured cells. Eur J Biochem 1979;93(1):51-55.

36. van den Boogert MAW, Rader DJ, Holleboom AG. New insights into the role of glycosylation in lipoprotein metabolism. Curr Opin Lipidol 2017;28(6):502-506.

37. Preston RJ, Rawley O, Gleeson EM, O'Donnell JS. Elucidating the role of carbohydrate determinants in regulating hemostasis: Insights and opportunities. Blood 2013;121(19):38013810 .

38. Kanai M, Akiyama M, Takahashi A, et al. Genetic analysis of quantitative traits in the japanese population links cell types to complex human diseases. Nat Genet 2018;50(3):390400.

39. Bowie A, Owens D, Collins P, Johnson A, Tomkin GH. Glycosylated low density lipoprotein is more sensitive to oxidation: Implications for the diabetic patient? Atherosclerosis 1993;102(1):63-67.

40. Cheng C, Ru P, Geng F, et al. Glucose-mediated N-glycosylation of SCAP is essential for SREBP-1 activation and tumor growth. Cancer Cell 2015;28(5):569-581.

41. Filipovic I. Effect of inhibiting N-glycosylation on the stability and binding activity of the low density lipoprotein receptor. J Biol Chem 1989;264(15):8815-8820.

42. Liu $\mathrm{D}$, Chu $\mathrm{X}$, Wang $\mathrm{H}$, et al. The changes of immunoglobulin $\mathrm{G} \mathrm{N-glycosylation} \mathrm{in} \mathrm{blood}$ lipids and dyslipidaemia. J Trans/ Med 2018;16(1):235-018-1616-2.

43. Teslovich TM, Musunuru K, Smith AV, et al. Biological, clinical and population relevance of 95 loci for blood lipids. Nature 2010;466(7307):707-713.

44. Nioi $P$, Sigurdsson $A$, Thorleifsson $G$, et al. Variant ASGR1 associated with a reduced risk of coronary artery disease. N Engl J Med 2016;374(22):2131-2141.

45. Menni C, Gudelj I, Macdonald-Dunlop E, et al. Glycosylation profile of immunoglobulin G is cross-sectionally associated with cardiovascular disease risk score and subclinical atherosclerosis in two independent cohorts. Circ Res 2018;122(11):1555-1564.

46. Harrus D, Khoder-Agha F, Peltoniemi M, et al. The dimeric structure of wild-type human glycosyltransferase B4GalT1. PLoS One 2018;13(10):e0205571.

47. Global Lipids Genetics Consortium, Willer CJ, Schmidt EM, et al. Discovery and refinement of loci associated with lipid levels. Nat Genet 2013;45(11):1274-1283. 


\section{FIGURES}

a

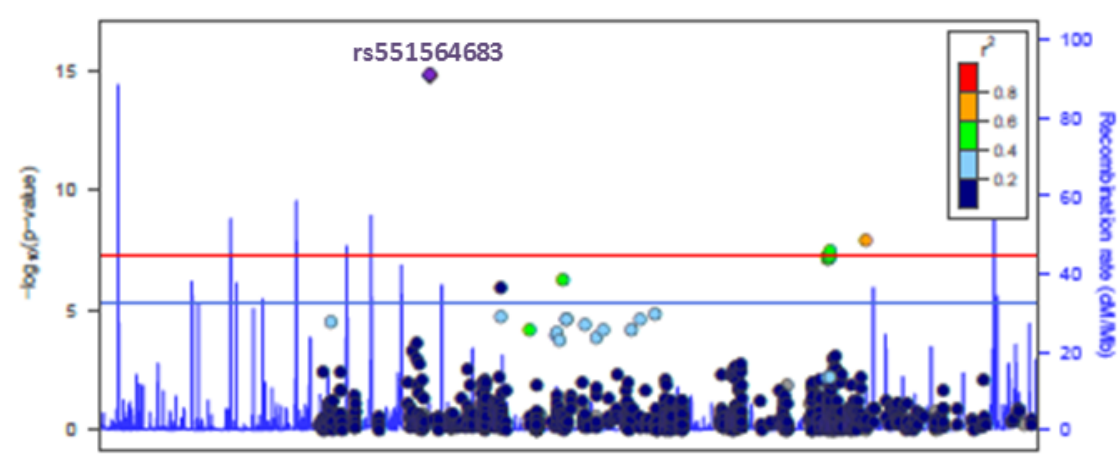

b

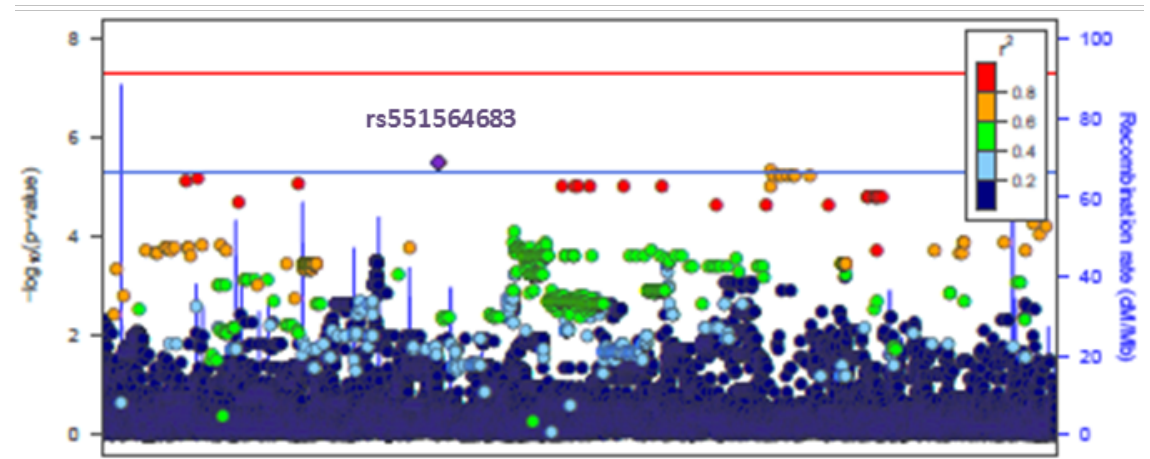

C

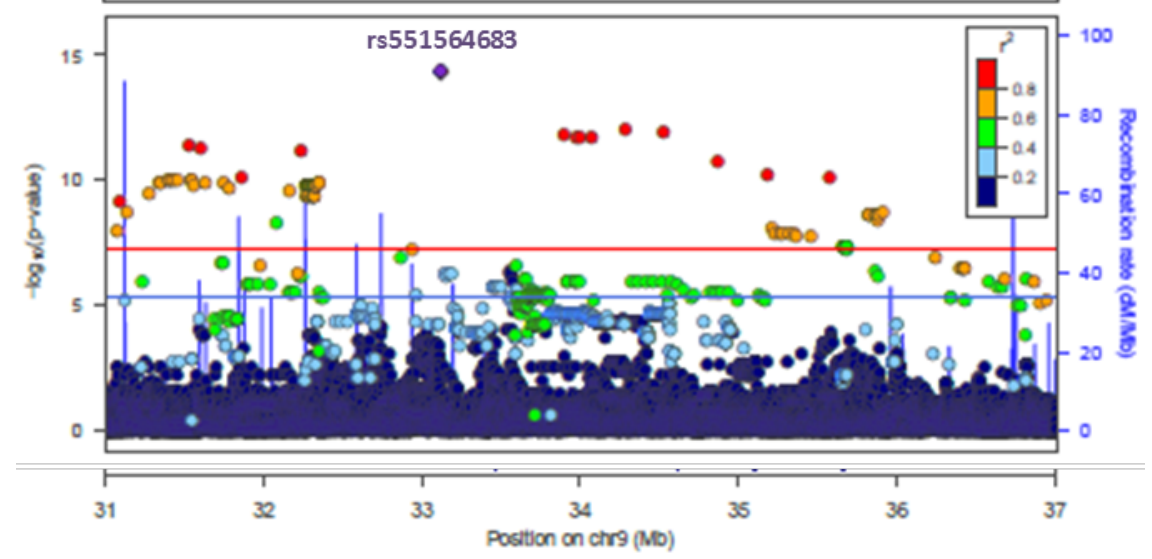

Fig. 1: Evidence for genetic association between rs551564683, encoding B4GALT1 p.Asn352Ser and LDL-C. a, Whole-exome sequencing results for 5,890 OOA subjects. b, Whole-genome sequencing results for 1,083 OOA subjects. c, Imputed data from genomewide genotyping chip results for 5,890 OOA subjects. Blue line marks a genome-wide suggestive threshold (5.0E-06) and red line a genome-wide significant threshold (5.0E-08). 


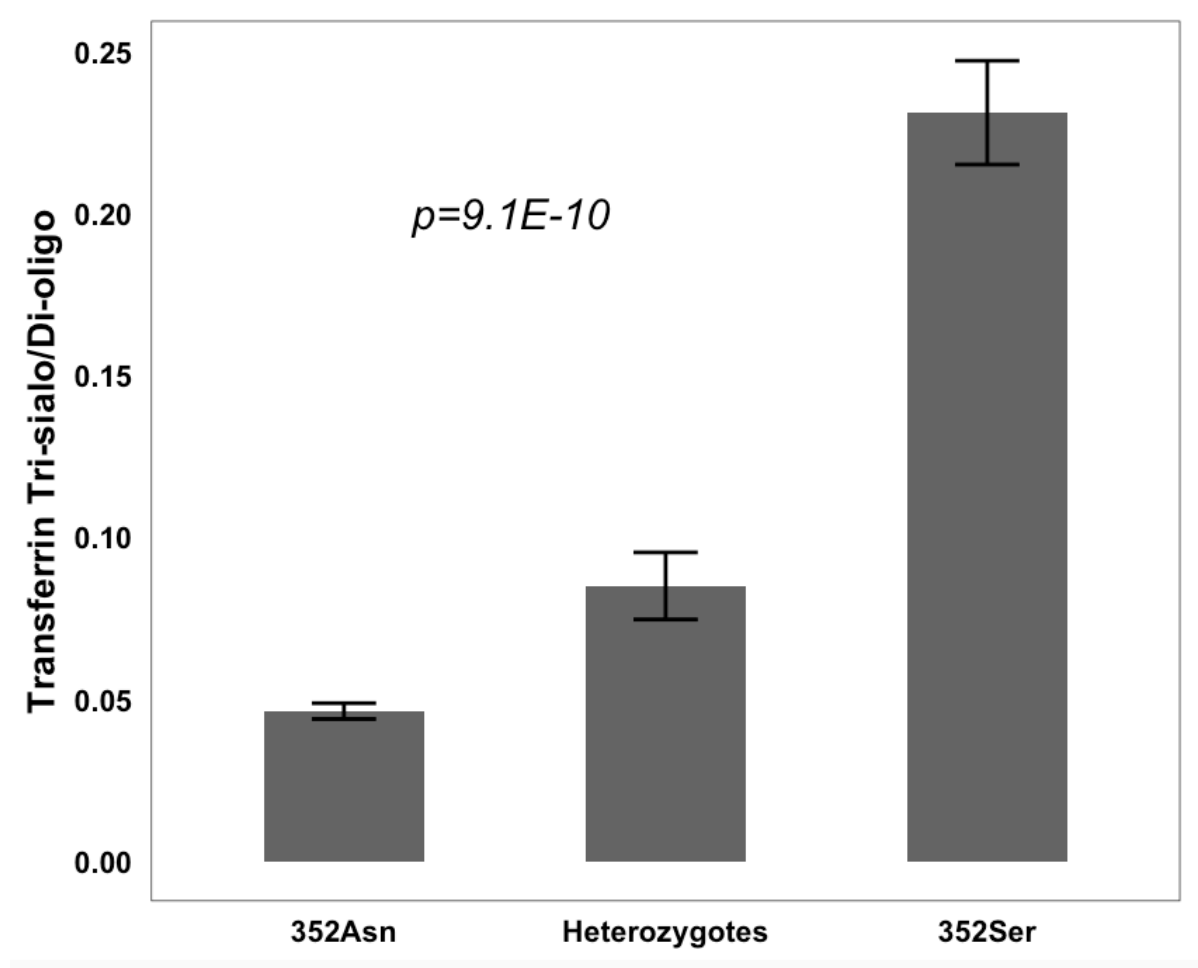

Fig. 2: B4GALT1 p.Asn352Ser is associated with increased carbohydrate-deficient transferrin. The transferrin Tri-sialo/Di-oligo ratio for the 3 genotype groups (11 352Asn homozygotes, 8 Asn352Ser heterozygotes and 9352 Ser homozygotes) as measured by the Carbohydrate Deficient Transferrin test. 


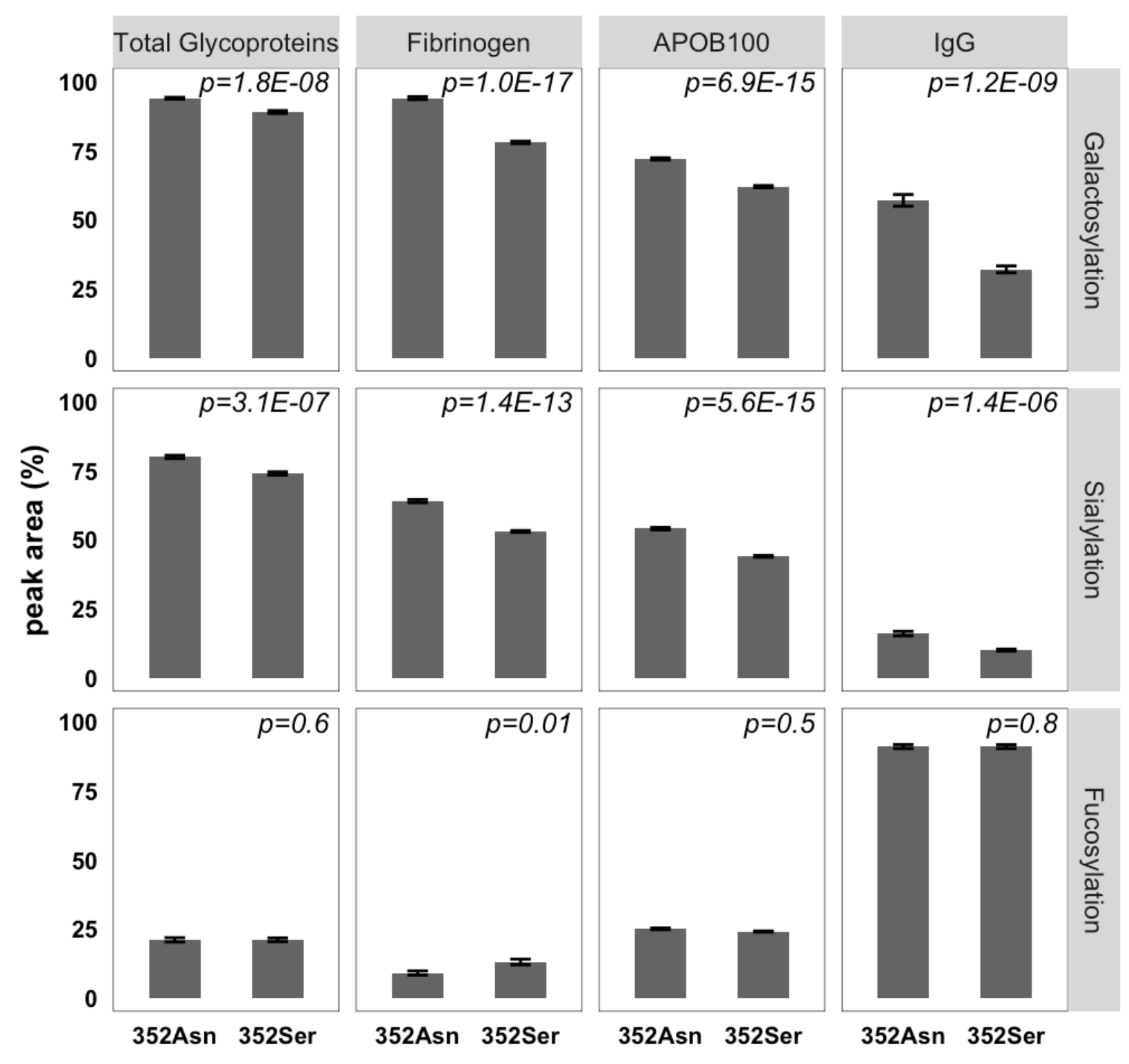

Fig. 3: B4GALT1 p.Asn352Ser is associated with decreased glycosylation. The levels of total galactosylation, sialylation and fucosylation in global plasma glycoproteins, fibrinogen, ApoB100, and IgG for 12 352Asn homozygotes and 12 352Ser homozygotes. Data are represented as mean and standard error. 


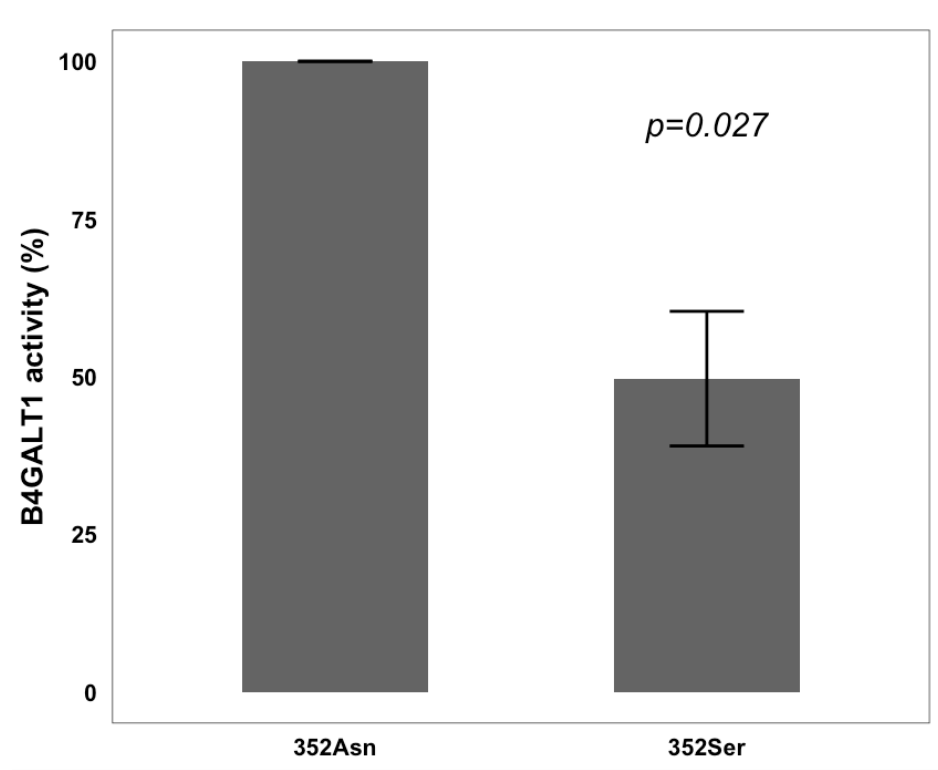

b

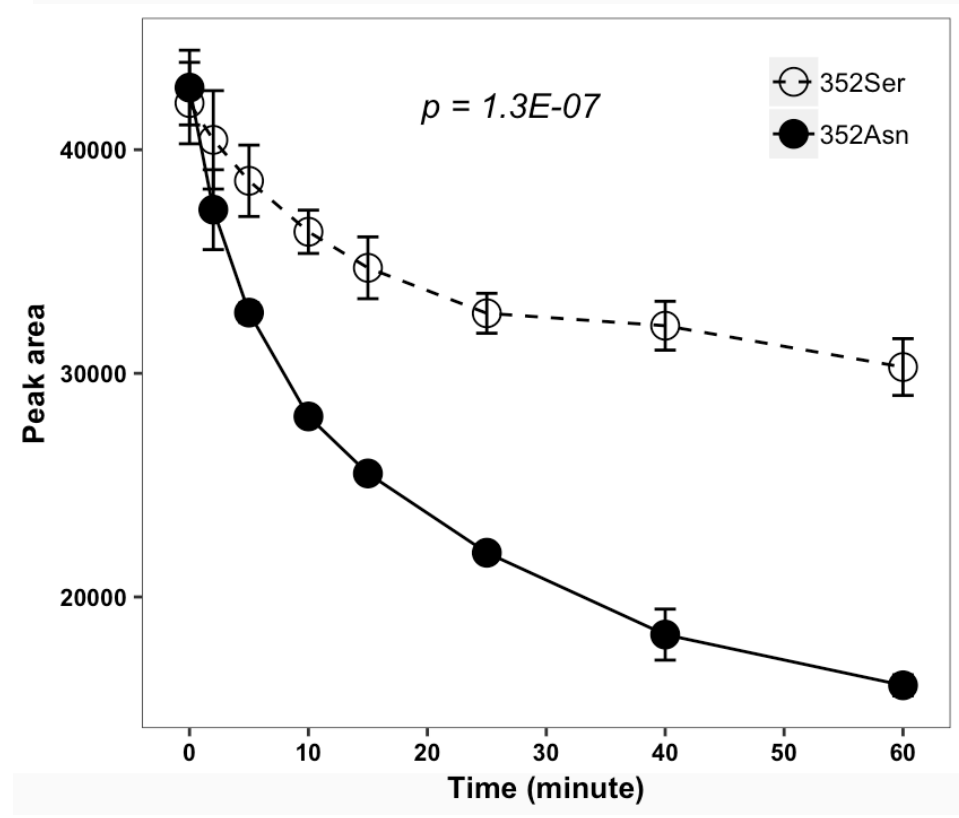

Fig. 4: B4GALT1 p.Asn352Ser decreases galactosyltransferase activity. a, The activity level of 352Asn B4GALT1 and 352Ser B4GALT1 immunoprecipitated proteins from transfected COS-7 cells. The data are expressed as the percent of 352Asn B4GALT1 activity and represent the mean of four experiments \pm the standard error. $\mathbf{b}$, The decrease in UDP-Gal incubated with recombinant B4GALT1 352Ser (open circles) and 352Asn proteins (solid circles). 


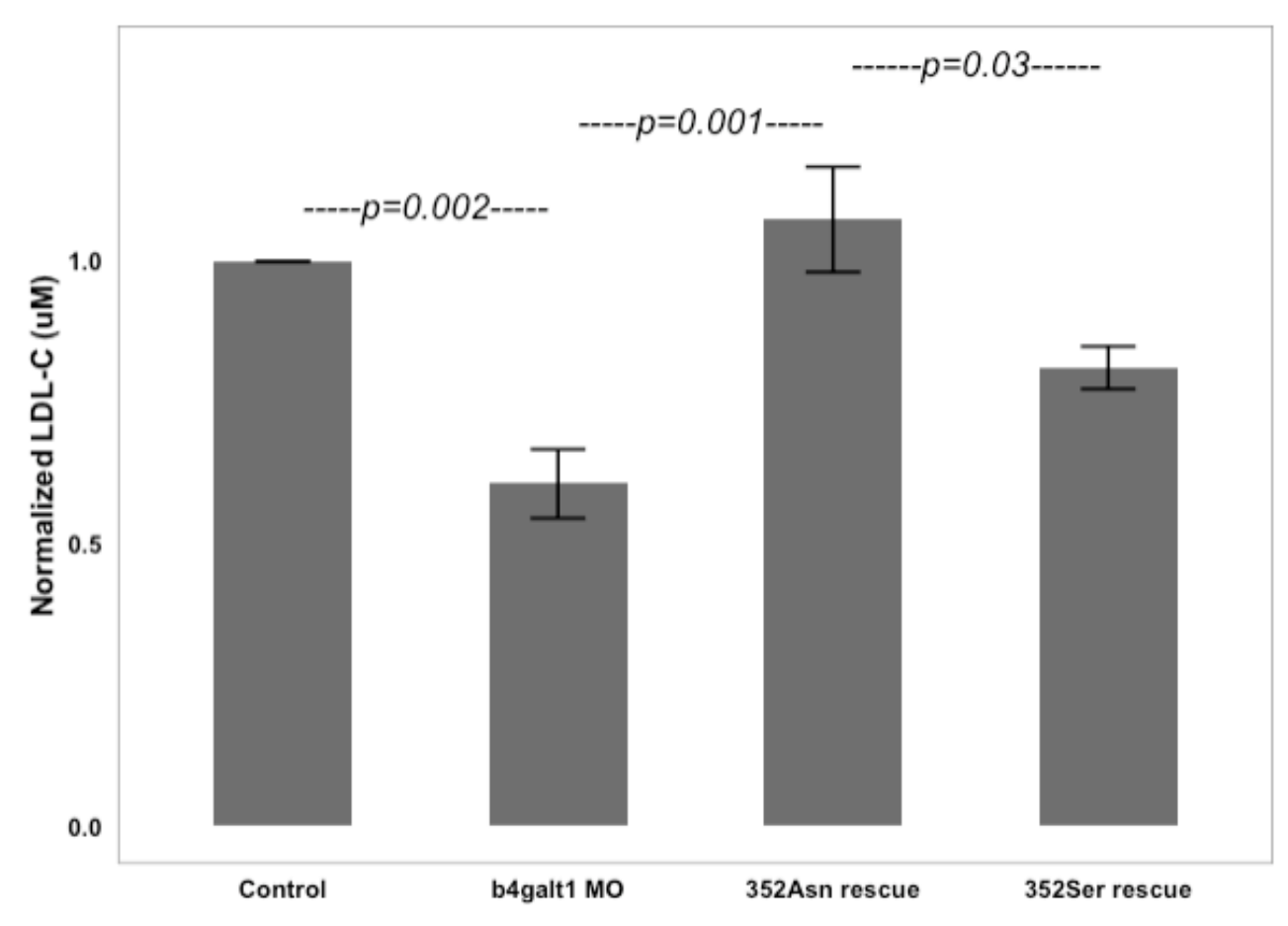

Fig. 5: Quantification of LDL-C in zebrafish. Average LDL-C in homogenates of unfed wild type $5 \mathrm{dpf}$ zebrafish larvae ( $\mathrm{n}=50$ per well, duplicated within each experiment, $n=7$ for Control, and 352Asn rescue, $n=5$ for b4galt1 $\mathrm{MO}, \mathrm{n}=4$ for 352 Ser rescue). Larvae were first injected with either a non-targeting control $\mathrm{MO}$ (Control) or $8 \mathrm{ng}$ of morpholino (b4galt1 $\mathrm{MO}$ ) against b4galt1 at 1-2 cell stage. Rescue conditions were $8 \mathrm{ng} \mathrm{MO}$ co-injected with 100pg 352Asn human B4GALT1 mRNA (352Asn rescue), or $8 \mathrm{ng} \mathrm{MO}$ co-injected with 100 pg B4GALT1 mRNA encoding the 352Ser mutation (352Ser rescue). Data are represented as mean and standard error, normalized to control $\mathrm{MO}$ within each experiment and averaged across experiments. 